\title{
Combined spinal-epidural anesthesia for lumbar discectomy in a patient with asymptomatic severe aortic stenosis -a case report-
}

\author{
Young Sung Kim, Ji Hye Park, Shin Young Lee, Byung Gun Lim, Heezoo Kim, Il-ok Lee, and \\ Myoung-Hoon Kong
}

Department of Anesthesiology and Pain Medicine, Korea University Guro Hospital, Seoul, Korea

The use of neuraxial anesthesia has traditionally been contraindicated in patients with severe aortic stenosis. However, general anesthesia can be riskier than neuraxial anesthesia for severe aortic stenosis patients undergoing spinal surgeries in the prone position as this can cause a major reduction in cardiac output secondary to diminished preload. In addition, general anesthesia, muscle relaxation, and positive-pressure ventilation can decrease venous return and reduce vascular tone, further compromising cardiac output. Combined spinal-epidural anesthesia with closely monitored, careful titration of the local anesthetic dose can be an efficient and safe anesthetic method for managing such patients. We describe the successful management of combined spinal-epidural anesthesia in an asymptomatic severe aortic stenosis patient scheduled for lumbar discectomy. (Korean J Anesthesiol 2014; 67: 129-132)

Key Words: Aortic stenosis, Combined spinal epidural anesthesia, Dexmedetomidine, Regional anesthesia.

Aortic stenosis (AS) is regarded as the most critical valvular lesion because of its potential to cause sudden death and the inability to obtain adequate systemic perfusion by external cardiac massage during cardiac arrest. A severe AS patient has multiple risk factors for anesthesiologists to consider, including severe hypotension, ischemic heart disease, and cerebral hypoperfusion. Generally, the use of neuraxial anesthesia (NA) is avoided in AS patients $[1,2]$ since the goal of anesthetic care is to increase left ventricular (LV) preload and systemic vascular resistance [3].

However, in some cases, general anesthesia (GA) can be riskier than NA for AS patients, such as those undergoing spinal surgeries performed in the prone position. The prone position can cause major reductions in cardiac output (CO) and stroke volume secondary to diminished preload and increased afterload [4,5], and these changes can cause significant hemodynamic compromise in AS patients with diminished cardiac reserve. Moreover, GA, muscle relaxation, and positive-pressure ventilation can interfere with venous return and reduce vascular tone; thus, further compromising the $\mathrm{CO}$, rendering AS patients vulnerable to uncompensated circulatory effects caused by

Received: June 11, 2013. Revised: June 24, 2013. Accepted: July 29, 2013.

Corresponding author: Byung Gun Lim, M.D., Ph.D., Department of Anesthesiology and Pain Medicine, Korea University Guro Hospital, 148, Gurodong-ro, Guro-gu, Seoul 152-703, Korea. Tel: 82-2-2626-1437, Fax: 82-2-851-9897, E-mail: bglim9205@korea.ac.kr (c) This is an open-access article distributed under the terms of the Creative Commons Attribution Non-Commercial License (http:// creativecommons.org/licenses/by-nc/3.0/), which permits unrestricted non-commercial use, distribution, and reproduction in any medium, provided the original work is properly cited. 
prone positioning.

Recently, an elderly patient with severe AS was scheduled for lumbar discectomy. To the best of our knowledge, the use of NA for patients with severe AS undergoing spinal surgeries has never been reported. We here describe the successful management of this patient with combined spinal-epidural anesthesia (CSEA) without any hemodynamic/neurologic complications or pain. The patient provided her written consent for the publication of this case.

\section{Case Report}

A 77-year-old woman (height, $155 \mathrm{~cm}$; weight, $50 \mathrm{~kg}$ ) with severe back pain and radiating pain in both legs was scheduled for lumbar discectomy under the diagnosis of L4-5 spinal stenosis. She had been able to perform basic activities of daily living despite a functional capacity of less than 4 metabolic equivalents and she had had no cardiac symptoms other than shortness of breath on exertion before the neurologic symptoms occurred. Upon preoperative evaluation, a 2-dimensional (2-D) echocardiography showed severe AS (aortic valve area $=0.79 \mathrm{~cm}^{2}$, pressure gradient $=88 / 53 \mathrm{mmHg}$ ) and concentric LV hypertrophy. However, no LV outlet obstruction and LV dilatation was observed and the LV contractility, stroke volume, and ejection fraction were normal. The necessity of cardiac surgery before lumber discectomy and the possibility that the lumbar surgery could be delayed were discussed with neurosurgeons and cardiologists taking into consideration the anesthetic and surgical risks. Considering the condition of her AS, the cardiac surgery should have been performed first. However, the patient refused the cardiac surgery for financial reasons and because of her fears relating to cardiac surgery. The lumbar surgery was required to prevent aggravation of her neurologic symptoms/deficits. Therefore, we decided that CSEA was the safest anesthetic method to use after considering the potential risks of GA that can occur in AS patients with diminished cardiac reserve (mentioned above), the patient's cardiac condition and functional capacity, and the characteristics of the operation.

On the day of surgery, the patient was very nervous despite being premedicated with intramuscular midazolam $2 \mathrm{mg}$; her blood pressure (BP) and heart rate (HR) were 160/70 $\mathrm{mmHg}$ and $95 \mathrm{bpm}$, respectively. Before performing anesthesia, the patient was sufficiently hydrated and elastic stockings were applied to both legs to minimize blood pooling. Electrocardiography, pulse oximetry, and continuous invasive arterial and central venous pressures were monitored. The $\mathrm{CO}$ was not monitored because noninvasive $\mathrm{CO}$ monitoring was not available in this regional anesthesia case and the Swan-Ganz catheter was considered too invasive for this case. Cardiac drugs including phenylephrine and epinephrine were kept ready. The patient received a rapid infusion of $500 \mathrm{ml}$ lactated Ringer's solution. Thereafter, her urinary bladder was catheterized and $2 \mathrm{~L} / \mathrm{min}$ of oxygen was administered via a nasal cannula. The patient was placed in the left lateral decubitus position, and after sterilization and numbing of the skin with local anesthesia, a 17-gauge Tuohy needle was placed at the L1-2 intervertebral space. After the epidural space was identified using the loss-of-resistance technique, a catheter was inserted $3 \mathrm{~cm}$ in the cephalad direction and a test dose of $2 \%$ lidocaine $3 \mathrm{ml}$ was injected via the catheter since neither blood nor cerebrospinal fluid was obtained on aspiration. Subsequently, a 25-gauge spinal needle was inserted at the L4-5 intervertebral space and $0.5 \%$ hyperbaric bupivacaine 8 mg was injected into the subarachnoid space. The patient was then immediately turned to a supine, reverse Trendelenburg position. Ten minutes later, the sensory block had reached the T11 dermatome, and her BP and HR were 145/70 mmHg and $90 \mathrm{bpm}$, respectively. Thereafter, $0.19 \%$ ropivacaine $8 \mathrm{ml}$ plus dexmedetomidine $50 \mu \mathrm{g}$ was divided in half and administered in separate doses through the epidural catheter with a 10 -min interval between doses. Twenty minutes later, the sensory block had reached the T9 dermatome, and her HR had decreased to 70 bpm, but her BP was maintained at 130-140/65-70 $\mathrm{mmHg}$. After she was turned to the prone position, the patient's BP and HR remained unchanged and surgery was initiated. The skin incision site was over the T10-11 dermatomes.

Throughout the surgery, the patient did not complain of pain and her vital signs were stable. The duration of the operation was $90 \mathrm{~min}$. Blood loss was minimal, and her urine output was about $350 \mathrm{ml} ; 850 \mathrm{ml}$ of crystalloid solution was administered. The central venous pressure was little changed during the perioperative period, and her cardiac enzymes levels were normal on postoperative day 1 . The patient was discharged without any hemodynamic instability or cardiovascular/neurologic complications on postoperative day 10 .

\section{Discussion}

Although the use of NA is traditionally contraindicated in AS patients, there have been a few reports of epidural or CSEA cases in which local anesthetics were slowly and gradually administered in an attempt to minimize hemodynamic change and prevent cardiovascular complications in such patients [6-8]. A previous report demonstrated the safety and effectiveness of hypotensive epidural anesthesia performed without any critical complications in 22 patients with noncritical asymptomatic AS who were undergoing total hip replacement surgery [9]. Considering hemodynamic stability, epidural anesthesia (EA) is preferred to spinal anesthesia (SA) due to the gradual onset of peripheral sympathetic nervous system blockade in AS patients. However, the neural blockade from EA is less intense than that 
from SA, and there is a greater chance of missed segments. Also, there has been 1 report of the successful application of continuous SA with a slow, gradual onset of neural blockade like that associated with EA in AS patients [10]. Therefore, we selected CSEA for the anesthesia of our patient since it has the advantages of both EA and SA.

GA was also an option for our patient. Although a previous study showed no difference in hemodynamics between CSEA and GA for renal transplant surgery [11], there is little evidence comparing the hemodynamics of CSEA and GA among AS patients. However, general anesthetics can depress the myocardium, produce vasodilatation, and can be associated with wide fluctuations in hemodynamics during periods of maximal (e.g., endotracheal intubation and extubation) and minimal stimulation. CSEA also produces vasodilatation below the level of the block, commonly resulting in hypotension, which may be compromising and can have negative effects in patients with severe AS. Therefore, with close monitoring, careful titration of the local anesthetic dose, and other adjunctive pharmacological support, CSEA was cautiously performed in our patient.

Conflicting results regarding mortality and morbidity rates have been reported in previous studies of non-cardiac surgeries for asymptomatic patients with severe AS. The reasons for these conflicting results are unclear, but maintenance of adequate ventricular volume and sinus rhythm is considered crucial for the management of AS patients [12]. We chose our anesthetic technique after carefully considering the patient's cardiac condition, functional capacity, and the characteristics of the operation to be performed.

First, we undertook a detailed evaluation of her cardiac condition and functional capacity. On the basis of her medical history and the results of the echocardiography evaluation, her AS was regarded as severe, but compensated, noncritical, and nearly asymptomatic.

Second, our patient was scheduled for lumbar discectomy, which requires that the patient be placed in the prone position for the surgical procedures. The prone position has measurable effects on the cardiovascular physiology of patients, especially a reduction in the cardiac index. This has been attributed to decreased venous return, direct effects on arterial filling, and reduced LV compliance secondary to increased thoracic pressure. Obstruction of the inferior vena cava is a well-known complication of prone positioning and is exacerbated by any degree of abdominal compression, leading to decreased $\mathrm{CO}$, venous stasis, and consequent thrombotic complications [5]. In these circumstances, GA, muscle relaxation, and positive-pressure ventilation should be avoided because they can further compromise the $\mathrm{CO}$, rendering AS patients vulnerable to adverse circulatory effects caused by prone positioning.

CSEA is usually performed simultaneously on the same spinal level. However, in this case, it was difficult to identify an appropriate position for the epidural catheter and to reach an adequate level of blockage because the skin incision site was on the T10-11 dermatomes, which is too far from the L4-5 intervertebral space. Moreover, if the epidural catheter was located on the L4-5 intervertebral space, which overlapped the surgical site, it may have interfered with the surgical procedure and increased the risk of surgical site infection. Therefore, we performed the EA on a different level (L1/2 intervertebral space) to that of the SA (L4-5 intervertebral space).

Dexmedetomidine is an alpha-2 adrenergic agonist that has an analgesic effect when used as an adjuvant in regional anesthesia [13]. A previous study showed that $1 \mu \mathrm{g} / \mathrm{kg}$ of dexmedetomidine provides an earlier onset and comparable analgesia with a lower consumption of local anesthetic as compared to $1 \mu \mathrm{g} / \mathrm{kg}$ of fentanyl when used as an adjuvant to local anesthetic in EA [14]. In our case, it was assumed that epidural dexmedetomidine might help CESA provide adequate analgesia, despite the use of a low concentration $(0.19 \%)$ of ropivacaine, and contribute to the maintenance of HR and BP within their normal ranges.

Continuous monitoring of intra-arterial BP and central venous and arterial filling pressure is mandatory for patients with severe AS $[9,10]$. In this case, although the patient underwent a single level discectomy, which is usually not accompanied by major fluid shifts, invasive monitoring of arterial and central vein pressure was performed to prevent any hemodynamic complications. Together, these strategies facilitated the successful management of our patient under CSEA without any hemodynamic/neurologic complications or pain.

In conclusion, we suggest that CSEA may be performed safely and effectively by using small, gradual, and incremental doses of local anesthetics for patients with asymptomatic, compensated severe AS undergoing lumbar surgery since this is of a short duration and without major fluid shifts. However, the use of NA remains controversial in other surgeries involving such patients. The choice of a suitable anesthetic technique for severe AS patients should be based on individualized assessments considering the cardiac condition on the basis of echocardiography findings, the type of surgery required and patient positioning, and the functional capacity of the patients.

\section{Acknowledgments}

This report was supported by a Korea University Grant to Dr. Lim. 


\section{References}

1. McDonald SB. Is neuraxial blockade contraindicated in the patient with aortic stenosis? Reg Anesth Pain Med 2004; $29: 496-502$.

2. Dawson JS. Epidurals in aortic stenosis. Reg Anesth Pain Med 2008; 33: 573.

3. Nussmeier NA, Hauser MC, Sarwar MF, Grigore AM, Searles BE. Anesthesia for cardiac surgical procedures. In: Miller's anesthesia. 7th ed. Edited by Miller RD: Philadelphia: Elsevier/Churchill Livingstone. 2010, pp 1932-3.

4. Dharmavaram S, Jellish WS, Nockels RP, Shea J, Mehmood R, Ghanayem A, et al. Effect of prone positioning systems on hemodynamic and cardiac function during lumbar spine surgery: an echocardiographic study. Spine (Phila Pa 1976) 2006; 31: 1388-93.

5. Edgcombe H, Carter K, Yarrow S. Anaesthesia in the prone position. Br J Anaesth 2008; 100: 165-83.

6. Xia VW, Messerlian AK, Mackley J, Calmes SH, Matevosian R. Successful epidural anesthesia for cesarean section in a parturient with severe aortic stenosis and a recent history of pulmonary edema--a case report. J Clin Anesth 2006; 18: 142-4.

7. Bundgaard-Nielsen M, Foss NB, Kristensen BB. Use of epidural blockade in a patient with hip fracture and aortic stenosis. Eur J Anaesthesiol 2005; 22: 471-2.

8. Boso EB. A case for combined spinal-epidural anesthesia for Cesarean section in a patient with aortic stenosis. W V Med J 2008; 104: 20-1.

9. Ho MC, Beathe JC, Sharrock NE. Hypotensive epidural anesthesia in patients with aortic stenosis undergoing total hip replacement. Reg Anesth Pain Med 2008; 33: 129-33.

10. Collard CD, Eappen S, Lynch EP, Concepcion M. Continuous spinal anesthesia with invasive hemodynamic monitoring for surgical repair of the hip in two patients with severe aortic stenosis. Anesth Analg 1995; 81: 195-8.

11. Urwin SC, Parker MJ, Griffiths R. General versus regional anaesthesia for hip fracture surgery: a meta-analysis of randomized trials. Br J Anaesth 2000; 84: 450-5.

12. Skubas NJ, Lichtman AD, Sharma A, Thomas SJ. Anesthesia for cardiac surgery. In: Clinical anesthesia. 6th ed. Edited by Barash PG, Cullen BF, Stoelting RK, Cahalan MK, Stock MC, Philadelphia: Lippincott Williams and Wilkins. 2009, pp 1078-80.

13. Bajwa SJ, Bajwa SK, Kaur J, Singh G, Arora V, Gupta S, et al. Dexmedetomidine and clonidine in epidural anaesthesia: A comparative evaluation. Indian J Anaesth 2011; 55: 116-21.

14. Bajwa SJ, Arora V, Kaur J, Singh A, Parmar SS. Comparative evaluation of dexmedetomidine and fentanyl for epidural analgesia in lower limb orthopedic surgeries. Saudi J Anaesth 2011; 5: 365-70. 